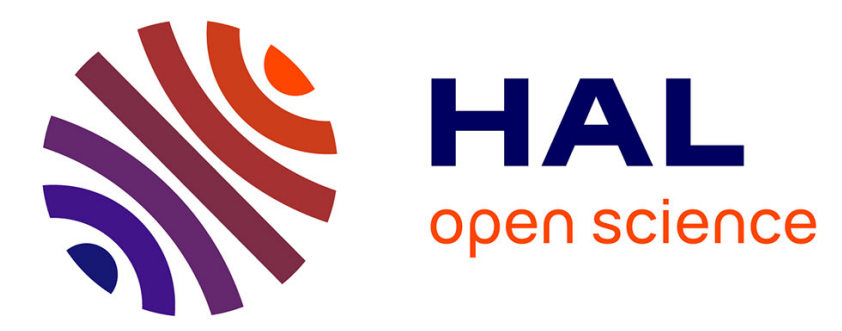

\title{
Comportement sous choc de composés lamellaires aluminium-cuivre
}

\author{
J.P. Romain, J. Jacquesson
}

\section{To cite this version:}

J.P. Romain, J. Jacquesson. Comportement sous choc de composés lamellaires aluminium-cuivre. Revue de Physique Appliquée, 1973, 8 (2), pp.157-163. 10.1051/rphysap:0197300802015700 . jpa00243671

\section{HAL Id: jpa-00243671 https://hal.science/jpa-00243671}

Submitted on 1 Jan 1973

HAL is a multi-disciplinary open access archive for the deposit and dissemination of scientific research documents, whether they are published or not. The documents may come from teaching and research institutions in France or abroad, or from public or private research centers.
L'archive ouverte pluridisciplinaire HAL, est destinée au dépôt et à la diffusion de documents scientifiques de niveau recherche, publiés ou non, émanant des établissements d'enseignement et de recherche français ou étrangers, des laboratoires publics ou privés. 
Classification

Physics Abstracts

$03.50-16.60$

\title{
COMPORTEMENT SOUS CHOC DE COMPOSÉS LAMELLAIRES ALUMINIUM-CUIVRE (*)
}

\author{
J. P. ROMAIN et J. JACQUESSON \\ Laboratoire de Mécanique et Physique des Matériaux \\ Groupe des Ondes de Choc \\ ENSMA, 2, rue Guillaume-VII, 86034 Poitiers, France \\ (Reçu le 13 octobre 1972, révisé le 19 janvier 1973)
}

\begin{abstract}
Résumé. - Des composés lamellaires aluminium-cuivre sont soumis à l'action d'ondes de choc planes se propageant dans une direction perpendiculaire au plan des lamelles. Leur comportement sous choc est étudié expérimentalement par une méthode de détection de choc originale utilisant l'effet électrique créé au passage d'une onde de choc à travers une jonction bimétallique. La stabilité du choc dans les composés étudiés est ainsi mise en évidence. On montre que ces composés peuvent être assimilés à des corps quasi homogènes dont on détermine les polaires de choc. La comparaison entre les courbes expérimentales et les courbes calculées selon le modèle adiabatique proposé par Tsou et Chou [1] montre un accord satisfaisant.
\end{abstract}

Abstract. - Plane shock waves are generated in lamellar aluminium copper composites. The direction of the wave propagation is perpendicular to the plane of the layers. The behavior under shock of these materials is experimentaly studied by means of an original shock detection technique based upon the electrical response under shock of a bimetallic junction. The shock front in these composites is found to be steady. It is shown that the composites behave like quasi homogeneous materials, the Hugoniot of which are determinated. The experimental Hugoniot curves compare satisfactorily with the calculated ones using the adiabatic model of Tsou and Chou [1].

1. Introduction. - Les propriétés de compressibilité dynamique des matériaux denses homogènes, en particulier des métaux, ont été abondamment étudiées et sont assez bien connues. Par contre, on possède encore peu de données sur le comportement sous choc des matériaux denses composites. L'étude de la compressibilité dynamique de tels matériaux s'est développée récemment [1] à [8]. Les travaux réalisés jusqu'ici ont porté principalement sur quelques matériaux lamellaires ou fibreux et sur des mélanges macroscopiquement isotropes. Sur le plan théorique, différents auteurs ont abordé le calcul de la polaire de choc d'un milieu hétérogène à partir des polaires de choc des constituants. Toutes les théories proposées supposent l'existence d'un choc stable dans le milieu considéré. La validité de cette hypothèse peut être vérifiée expérimentalement, comme l'ont fait par exemple Holmes et Tsou [8] sur un composé fibreux.

Nous présentons ici les résultats d'une étude expérimentale sur des composés lamellaires bimétalliques

(*) Etude effectuée sous contrat du Commissariat à l'Energie Atomique, avec le concours des Centres d'Etudes du Ripault et de Vaujours. aluminium-cuivre, soumis à l'action de chocs plans se propageant dans une direction perpendiculaire au plan des lamelles. On étudie la stabilité du choc induit dans ces composés par un procédé original utilisant l'effet électrique dû au passage d'une onde de choc sur une jonction bimétallique [9], [10], [11]. A partir des mesures de vitesse de choc, on détermine les polaires de choc des matériaux étudiés. Ces polaires sont ensuite comparées aux polaires calculées à partir des propriétés des constituants selon le modèle adiabatique de Tsou et Chou [1].

2. Matériaux étudiés. Dispositif expérimental. 2.1 MatériauX ÉTUDIÉS. - Le choix de composés $\mathrm{Al}-\mathrm{Cu}$ a été motivé essentiellement par les raisons suivantes : le cuivre et l'aluminium servent très souvent de matériaux de référence dans les études de compression par onde de choc; leur comportement est bien connu et ne présente pas d'anomalies (changements de phase par exemple) ; enfin, les compressibilités de ces deux métaux sont assez différentes pour que l'influence de leurs proportions relatives sur l'évolution de la compressibilité des composés $\mathrm{Al}-\mathrm{Cu}$ puisse être étudiée de façon significative. 
Le choix de structures lamellaires repose sur la facilité de réalisation de telles structures sous forme d'empilements de lamelles des métaux constituants. En outre, l'épaisseur des lamelles est un paramètre que l'on peut aisément faire varier pour étudier son influence éventuelle sur la compressibilité du composé. Enfin, ce type de structure peut donner lieu à des formulations théoriques simplifiées par rapport à des structures moins ordonnées.

Les échantillons étudiés ici sont constitués par des empilements de lamelles alternativement en cuivre et en aluminium, pressées les unes sur les autres de manière à réaliser un milieu continu. On distinguera deux séries d'échantillons caractérisées par des compositions massiques globales différentes : série $E$ de composition $33 \% \mathrm{Cu}-67 \% \mathrm{Al}$ et série I de composition $45 \% \mathrm{Cu}-55 \% \mathrm{Al}$. La série I est divisée en trois groupes désignés par $I_{1}, I_{2}, I_{3}$ et différant par l'épaisseur et le nombre des lamelles constituant les échantillons.

Le tableau I résume les caractéristiques de ces différents types d'échantillons.

L'épaisseur globale des échantillons est mesurée après serrage, la valeur obtenue s'écarte quelquefois légèrement de la valeur prévue et indiquée dans le tableau I. Ceci est attribué d'une part à des fluctuations de faible amplitude dans l'épaisseur des feuilles de métal d'où sont tirées les lamelles, et d'autre part à un mattage des lamelles lors du montage des essais. La composition globale définitive est calculée en tenant compte de cet effet et à partir des masses volumiques de l'aluminium et du cuivre, respectivement $2,70 \mathrm{~g} / \mathrm{cm}^{3}$ et $8,93 \mathrm{~g} / \mathrm{cm}^{3}$.

Les échantillons ainsi constitués sont soumis à l'action d'ondes de choc planes engendrées par des explosifs liquides ou solides. Le schéma du dispositif expérimental utilisé est représenté figure 1 . Le générateur de choc est appliqué sur une plaque de cuivre ou d'aluminium d'épaisseur variable. L'empilement des lamelles est pressé sur la face opposée de cette plaque dont le rôle est de transmettre et amortir le choc créé par le générateur explosif. L'amplitude du choc transmis dans l'échantillon dépend donc à la fois de l'épaisseur de la plaque amortisseur et de la nature de l'explosif utilisé.

Pour chaque expérience, la pression génératrice de référence désignée par $P_{\mathrm{R}}$ est celle de l'onde de choc incidente à sa sortie de la plaque amortisseur. Les valeurs de $P_{\mathrm{R}}$ ont été déterminées en fonction de l'épais-

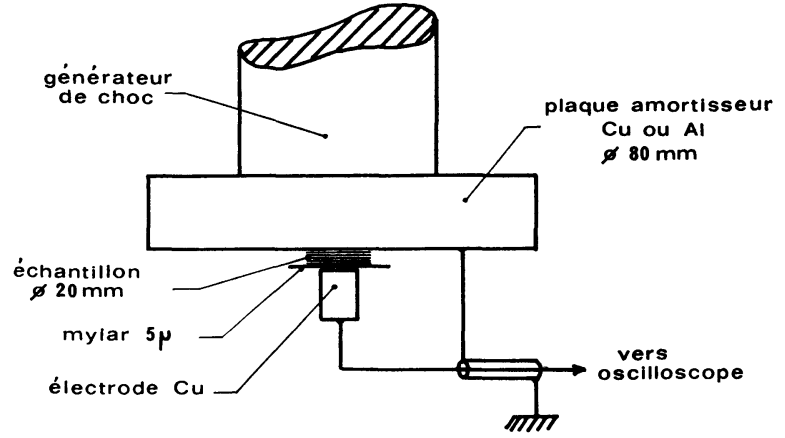

FIG. 1. - Schéma du dispositif expérimental.

seur de la plaque par un étalonnage préalable. La reproductibilité du choc a été vérifiée. La dispersion est de quelques kbars seulement, ce qui n'introduit pas d'erreur notable dans la détermination des courbes de compressibilité dynamique des corps étudiés.

La propagation du choc dans l'échantillon lamellaire est visualisée par enregistrement à l'oscilloscope des impulsions électriques créées au passage de l'onde de choc à travers les interfaces bimétalliques $\mathrm{Al}-\mathrm{Cu}$ successifs. L'amplitude de ces impulsions est, pour le couple $\mathrm{Al}-\mathrm{Cu}$ et les pressions utilisées ici, de l'ordre de $10 \mathrm{mV}$. L'instant d'arrivée de l'onde de choc à l'extrémité de l'échantillon est repéré avec précision grâce à une feuille de mylar d'épaisseur $5 \mu$ introduite entre la dernière lamelle de l'empilement et l'électrode poussoir. La polarisation du mylar sous choc produit une impulsion à front raide de l'ordre de $100 \mathrm{mV}$, très nettement différentiable des impulsions dues à l'effet de jonction $\mathrm{Al}-\mathrm{Cu}$. La figure 2 reproduit un oscillogramme typique obtenu avec ce dispositif. En

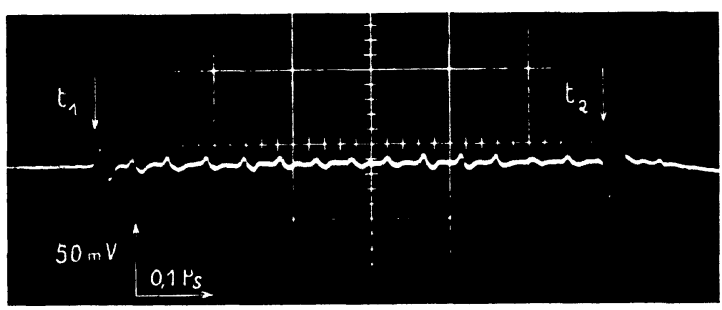

FIG. 2. - Oscillogramme typique. Composé Al-Cu type $\mathrm{I}_{3}$. $P_{\mathrm{R}}=107 \mathrm{kbar}(\mathrm{Al})$.

$t_{1}=$ arrivée de l'onde de choc sur la première lamelle de l'empilement.

$t_{2}=$ sortie de l'onde de choc de l'échantillon (impulsion mylar).

TABleaU I

Composition et structure des échantillons

Type d'échantillon

Composition massique globale

Masse volumique globale $\left(\mathrm{g} / \mathrm{cm}^{3}\right)$

Epaisseur des lamelles de $\mathrm{Cu}(\mathrm{mm})$

Epaisseur des lamelles de $\mathrm{Al}$ (mm)

Nombre de couples de lamelles Al-Cu

Epaisseur totale de l'échantillon ( $\mathrm{mm}$ )

$\begin{array}{ccc}\mathrm{E} & \mathrm{I}_{1} \\ 33 \% \mathrm{Cu}-67 & \% \mathrm{Al} & \\ 3,51 & & \\ 0,03 & & 0,03 \\ 0,20 & 0,12 \\ 8 & 14 \\ 1,84 & & 2,10\end{array}$

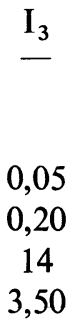


comparaison des autres systèmes de détection électriques ou optiques couramment utilisés pour ce genre d'expérience, ce procédé présente l'avantage de pouvoir visualiser la progression du choc au sein même du corps étudié et de permettre ainsi une étude chronométrique précise du phénomène. De plus, l'échantillon constituant son propre capteur, aucune perturbation n'est introduite dans la propagation du choc par le système de détection.

3. Résultats expérimentaux. Assimilation du milieu hétérogène à un milieu homogène. - Le comportement sous choc du composé $\mathrm{E}$ et des différentes formes du composé I a été étudié à l'aide du procédé décrit ci-dessus. Les résultats obtenus portent sur la stabilité du choc, la détermination des courbes de compressibilité dynamique des composés et l'existence pour ces composés d'une relation linéaire entre vitesse de choc et vitesse particulaire.

3.1 Stabilité Du choc. - Les diagrammes de marche du front de choc dans les échantillons ont été construits à partir de la détermination sur les oscillogrammes des instants de traversée des interfaces $\mathrm{Al}-\mathrm{Cu}$. La figure 3 en donne des exemples pour les différents types de composés. Il apparaît qu'aux erreurs de mesure près, la vitesse de l'onde de choc reste constante pendant toute la traversée de l'échantillon. L'épaisseur totale d'un échantillon est déterminée à $0,01 \mathrm{~mm}$ près, et l'incertitude sur chaque mesure de temps est de 5 ns.

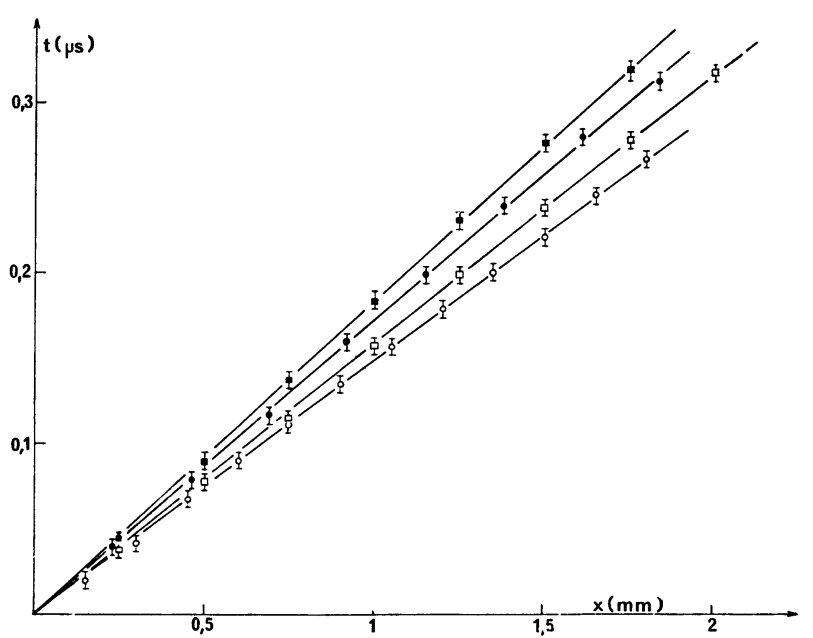

Fig. 3. - Diagrammes de marche du front d'onde de choc.

$$
\begin{aligned}
& \text { - Composé de type E, } P_{\mathrm{R}}=87 \mathrm{kbar} \text { (Al). } \\
& \text { ○ - - } \quad \mathrm{I}_{1}, \quad P_{\mathrm{R}}=310 \mathrm{kbar}(\mathrm{Al}) \text {. } \\
& \text { - } \quad-\quad \text { - } \mathbf{I}_{2}, \quad P_{\mathrm{R}}=97 \mathrm{kbar}(\mathrm{Al}) \text {. } \\
& \square \quad-\quad-\quad \mathrm{I}_{3}, \quad P_{\mathrm{R}}=243 \mathrm{kbar}(\mathrm{Al}) \text {. }
\end{aligned}
$$

La constitution lamellaire des échantillons donne lieu à des phénomènes de transmissions et de réflexions d'ondes multiples. Une onde de choc stable, dans un tel milieu, ne peut donc résulter que de l'établissement progressif d'un état de régime. La vitesse du choc apparaissant constante pendant toute la traversée de l'échantillon, on en déduit que cet état stable de régime est très rapidement atteint. Cependant, compte tenu de la précision des mesures, une faible variation de la vitesse de choc sur une distance très courte ne peut être mise en évidence. Entre deux couples de lamelles consécutifs, par exemple, seules des variations de vitesse supérieures à $10 \%$ peuvent produire un écart significatif. La linéarité des diagrammes montre néanmoins que la traversée de quelques lamelles suffit pour atteindre l'état stationnaire. La vitesse du choc est déterminée à partir du temps mis par l'onde pour traverser complètement l'échantillon avec une précision de $2 \%$.

Une transmission simple du front initial, négligeant l'influence d'ondes arrières issues de réflexions secondaires sur les interfaces $\mathrm{Al}-\mathrm{Cu}$, donnerait, d'après le calcul et selon la pression, une vitesse moyenne de 2 à $7 \%$ inférieure aux valeurs expérimentales. L'apport d'énergie dû à ces ondes arrières et leur rôle dans l'établissement $\mathrm{du}$ front stationnaire, apparaissent donc importants et ne peuvent être négligés.

3.2 Polaires De CHOC. - La stabilité du choc dans les composés lamellaires étudiés montre que le comportement de ces milieux est assimilable à celui de corps homogènes. Les lois de conservation de la masse de la quantité de mouvement et de l'énergie à travers le choc sont donc applicables. Connaissant la valeur $P_{\mathrm{R}}$ de la pression incidente de référence dans la plaque amortisseur ainsi que la vitesse $D$ du choc induit dans le milieu étudié, on en déduit, par la méthode des impédances non adaptées [12], la pression $P$ ainsi que la vitesse particulaire $u$, derrière le choc dans le milieu équivalent au composé. Les valeurs expérimentales de ces paramètres figurent dans les tableaux II et III. On y a reporté également les valeurs de la masse volumique initiale $\rho_{0}$ de chaque échantillon, et de la masse volumique $\rho$ dans l'état comprimé, calculée à partir de $\rho_{0}$ en appliquant les lois de conservation à travers le choc. L'état hydrodynamique du composé est ainsi complètement déterminé. La représentation de cet état et son évolution dans le plan $(P, u)$ constituent la polaire de choc du milieu.

Sur la figure 4 sont reproduites les polaires des composés étudiés ainsi que, en comparaison, celles $\mathrm{du}$ cuivre et de l'aluminium. On constate que les courbes relatives aux différentes formes du composé I sont confondues.

L'unicité des polaires relatives aux types $\mathrm{I}_{2}$ et $\mathrm{I}_{3}$ résulte de la stabilité du choc dans le milieu lamellaire. En effet, les échantillons de ces deux types ne diffèrent que par leur épaisseur totale ; comme l'onde de choc est stable dès le début de sa propagation dans l'échantillon, sa vitesse ne dépend donc pas de l'épaisseur traversée.

Les échantillons de type $I_{1}$ et $I_{2}$ diffèrent uniquement par l'épaisseur des lamelles de cuivre et d'aluminium qui les constituent. La coïncidence des polaires des 
TABleau II

Résultats expérimentaux : Composé Al-Cu type E $33 \% \mathrm{Cu}-67 \% \mathrm{Al}$

$\begin{gathered}\beta_{0} \\ \left(\mathrm{~g} / \mathrm{cm}^{3}\right)\end{gathered}$
-
3,52
3,52
3,52
3,52
3,52
3,52
3,50
3,52
3,52
3,52
3,51
3,49

\begin{tabular}{|c|}
\hline$\underset{\text { (kbar) }}{P_{\mathrm{R}}}$ \\
\hline 77 (Al) \\
\hline 77 (Al) \\
\hline 87 (Al) \\
\hline 97 (Al) \\
\hline 107 (Al) \\
\hline 107 (Al) \\
\hline $117(\mathrm{Al})$ \\
\hline 243 (Al) \\
\hline 243 (Al) \\
\hline 284 (Al) \\
\hline $332(\mathrm{Cu})$ \\
\hline $360(\mathrm{Cu})$ \\
\hline
\end{tabular}

$D$
$(\mathrm{~mm} / \mu \mathrm{s})$
-
5,55
5,54
5,47
5,81
5,73
5,57
5,87
6,55
6,55
6,75
6,20
6,37

$P$
(kbar)
-
84
84
94
108
116
114
132
269
269
312
212
230

$u$
$(\mathrm{~mm} / \mu \mathrm{s})$
-
0,430
0,430
0,490
0,530
0,570
0,580
0,640
1,170
1,170
1,315
0,970
1,035

$\left.\stackrel{\rho}{\rho} \mathrm{cm}^{3}\right)$

3,815

3,816

3,866

3,873

3,908

3,929

3,928

4,285

4,285

4,370

4,161

4,167

TABLEAU III

Résultats expérimentaux composé Al-Cu type I $45 \% \mathrm{Cu}-55 \% \mathrm{Al}$

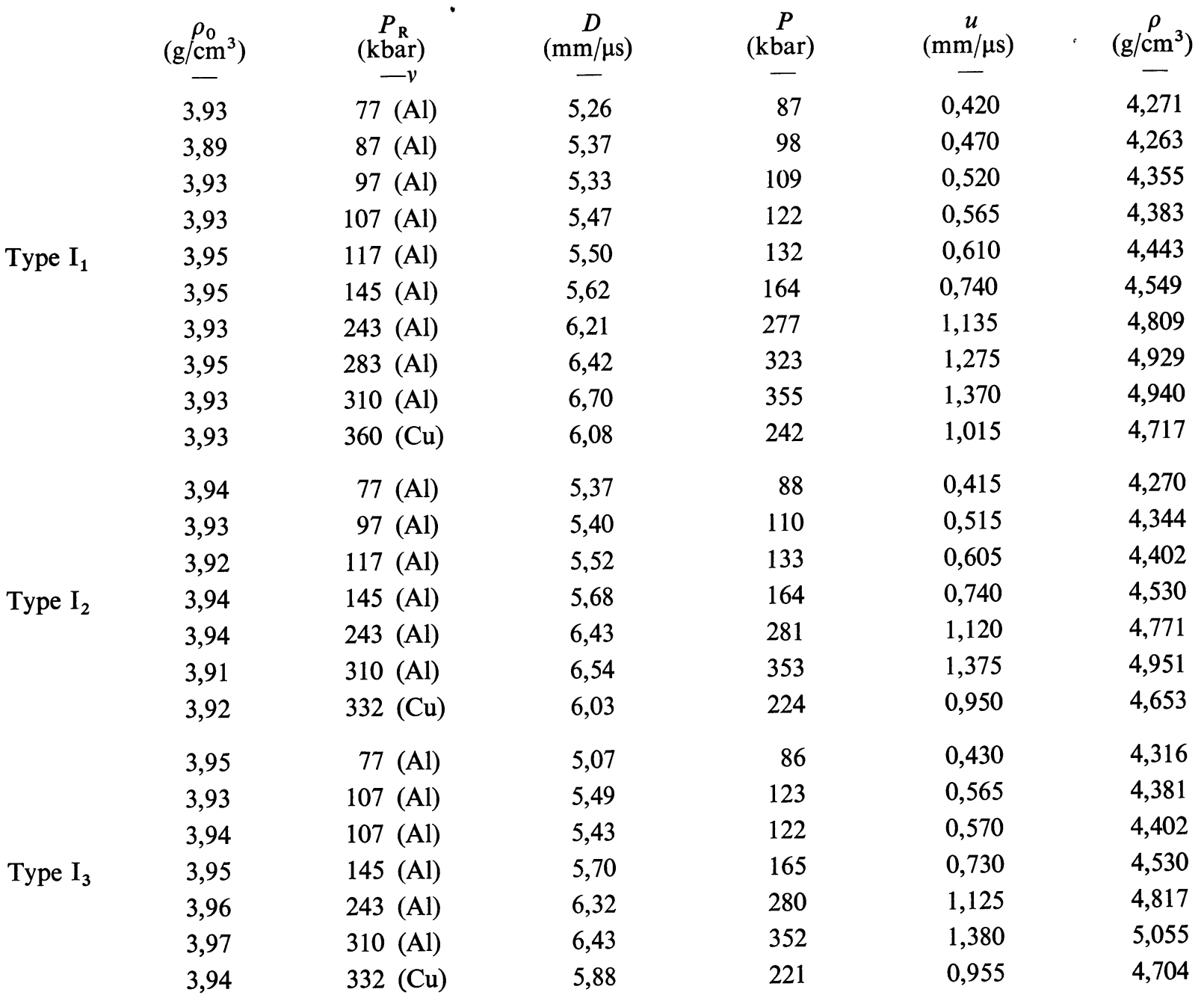




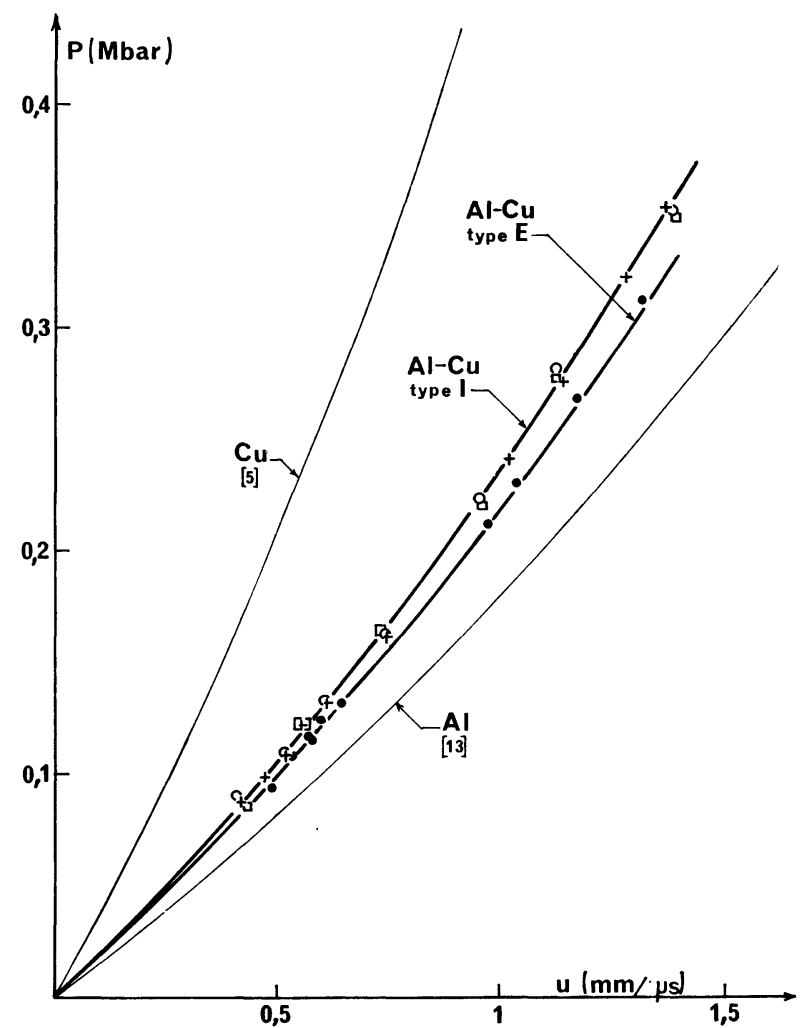

Fig. 4. - Polaire de choc des composés lamellaires $\mathrm{Al}-\mathrm{Cu}$, Résultats expérimentaux : $\bigcirc$ type $E,+$ type $I_{1}, O$ type $I_{2}$. type $\mathbf{I}_{3}$.

types $I_{1}$ et $I_{2}$ montre donc que l'épaisseur des lamelles, c'est-à-dire la dimension des hétérogénéités des milieux étudiés, n'a pas d'influence sur les propriétés de compressibilité dynamique globale de ces milieux. Ce résultat légitime l'assimilation des composés lamellaires à des milieux quasi homogènes.

3.3 RELATION LINÉAIRE ENTRE VITESSE DE CHOC ET VITESSE PARTICULAIRE. - Une autre représentation usuelle de l'état hydrodynamique d'un milieu soumis à l'action d'une onde de choc peut se faire dans le plan $(D, u)$. Dans le cas de corps homogènes isotropes la relation expérimentale entre $D$ et $u$ est linéaire avec une excellente approximation dans le domaine de pression étudié ici [12], [14].

$$
D=C+S u \text {. }
$$

Les polaires de référence du cuivre et de l'aluminium correspondent aux relations linéaires suivantes entre vitesse de choc et vitesse particulaire exprimées en $\mathrm{mm} / \mu \mathrm{s}$.

Pour le cuivre $\quad D=3,94+1,489 u$ d'après [5].

Pour l'aluminium $D=5,38+1,337 u$ d'après [13].

La représentation des résultats expérimentaux relatifs à nos composés dans le plan $(D, u)$, figure 5 , montre que les points se placent avec une bonne approximation sur deux droites : l'une correspond au composé $\mathrm{E}$ et l'autre correspond à l'ensemble des trois formes

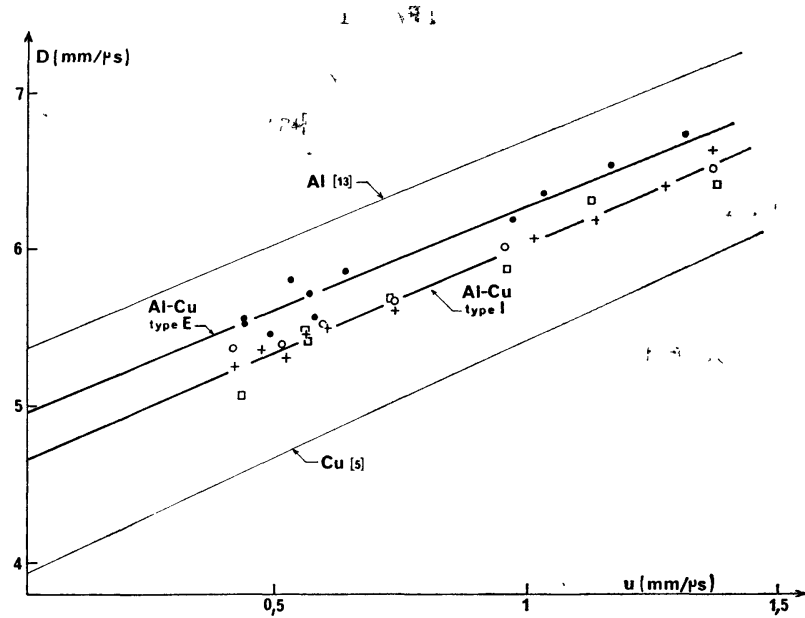

Fig. 5. - Relations linéaires $D=C+S u$. Résultats expérimentaux :- type $\mathrm{E},+$ type $\mathrm{I}_{1}, \bigcirc$ type $\mathbf{I}_{2}, \square$ type $\mathbf{I}_{3}$.

du composé I, conformément à l'identité de comportement observée plus haut. Ces relations linéaires confirment l'équivalence de nos milieux hétérogènes lamellaires à des milieux homogènes. La position des droites, déterminée par la méthode des moindres carrés correspond aux équations suivantes où $D$ et $u$ sont exprimées en $\mathrm{mm} / \mu \mathrm{s}$.

Pour le composé E : $D=4,97+1,304 u$.

Pour le composé I : $D=4,67+1,374 u$.

L'écart type des valeurs expérimentales de $D$ à la droite des moindres carrés est :

Pour le composé E : $\sigma_{\mathrm{E}}=0,084 \mathrm{~mm} / \mu \mathrm{s}$.

Pour le composé I : $\sigma_{\mathrm{I}}=0,087 \mathrm{~mm} / \mu \mathrm{s}$.

La valeur du premier coefficient $C$, extrapolation de la relation linéaire à vitesse particulaire nulle, doit correspondre à la valeur $C_{\mathrm{B}}$ de la vitesse du son hydrodynamique (Bulk sound velocity). Les valeurs de $C$ obtenues expérimentalement peuvent être comparées aux valeurs de $C_{\mathrm{B}}$ que l'on peut calculer à partir des propriétés élastiques des constituants. En effet, dans un milieu lamellaire, lorsque la direction de propagation des ondes est perpendiculaire au plan des lamelles, le temps mis par une onde élastique pour traverser un nombre donné de lamelles, est simplement la somme des temps de traversée de chacune des lamelles, c'est-à-dire, pour un composé à $n$ constituants.

$$
\frac{1}{C_{\mathrm{B}}}=\sum_{i=1}^{n} \frac{\gamma_{i}}{C_{\mathrm{B} i}}
$$

où $C_{\mathrm{B} i}$ représente la vitesse $\mathrm{du}$ son hydrodynamique dans le $i$-ième constituant dont $\gamma_{i}$ est la fraction volumique.

Nous avons déterminé $C_{\mathrm{B}}$ pour le cuivre et l'aluminium d'après leurs propriétés élastiques tirées de [15]. L'application de la relation précédente donne $C_{\mathrm{B}}$ pour les composés $\mathrm{E}$ et $\mathrm{I}$. Ces différentes valeurs sont portées dans le tableau IV. On constate alors que l'écart entre ces valeurs et celles de $C$ est du même 
TABLEAU IV

\begin{tabular}{lccc}
\multicolumn{1}{c}{} & $\begin{array}{c}C \\
(\mathrm{~mm} / \mu \mathrm{s})\end{array}$ & $\begin{array}{c}C_{\mathrm{B}} \\
(\mathrm{mm} / \mu \mathrm{s})\end{array}$ & Ecart relatif \\
Aluminium & - & - & - \\
Cuivre & 5,38 & 5,23 & $2,9 \%$ \\
Composé E & 3,94 & 3,87 & $1,8 \%$ \\
Composé I & 4,97 & 5,00 & $0,6 \%$ \\
& 4,67 & 4,89 & $4,5 \%$
\end{tabular}

ordre de grandeur pour les composés et pour les métaux purs. Ceci confirme à nouveau la validité de la relation linéaire entre vitesse de choc et vitesse particulaire obtenue pour les composés lamellaires assimilés à des milieux continus. On pourra donc ne pas faire intervenir la dimension des hétérogénéités pour étudier la compressibilité de tels milieux. Seule la composition est importante.

4. Comparaison entre courbes de compressibilité expérimentales et courbes théoriques. - Plusieurs théories ont été proposées pour calculer la courbe de compressibilité dynamique d'un composé à partir de sa composition et des courbes de Hugoniot de ses constituants. Ces théories diffèrent par les hypothèses d'interactions entre constituants sous l'action du choc. Certaines d'entre elles conduisent à des résultats très voisins, comme l'ont montré Garg et Kirsch [7] dans une étude comparative des différentes méthodes.

Pour calculer les courbes de compressibilité dynamique, ou courbes de Hugoniot, de nos composés Al$\mathrm{Cu}$, nous avons choisi la méthode développée par Tsou et Chou [1] supposant que l'équilibre des pressions et des vitesses matérielles se réalise de manière adiabatique. Pour ce calcul, on doit connaître l'équation d'état de chaque constituant, que Tsou et Chou expriment sous la forme :

$$
\begin{aligned}
P=A\left(\frac{\rho}{\rho_{0}}-1\right)+B\left(\frac{\rho}{\rho_{0}}-1\right)^{2} & + \\
& +D\left(\frac{\rho}{\rho_{0}}-1\right)^{3}+G \rho E
\end{aligned}
$$

où $P$ est la pression, $\rho$ la masse volumique, $\rho_{0}$ la masse volumique à la pression initiale, cette dernière étant négligeable devant la pression atteinte dans le choc. $E$ représente l'énergie interne spécifique. $A, B, D, G$ sont des coefficients dont les valeurs, indiquées dans le tableau $\mathrm{V}$, ont été calculées pour l'aluminium et le

\section{TABleaU $\mathrm{V}$}

$\begin{array}{lccccc} & \begin{array}{c}\rho_{0} \\ \left(\mathrm{~g} / \mathrm{cm}^{3}\right)\end{array} & \begin{array}{c}A \\ (\mathrm{Mbar})\end{array} & \begin{array}{c}B \\ (\mathrm{Mbar})\end{array} & \begin{array}{c}D \\ (\mathrm{Mbar})\end{array} & G \\ \mathrm{Al} & - & - & - & - & - \\ \mathrm{Cu} & 2,70 & 0,765 & 0,844 & -1,339 & 2,13 \\ & 8,93 & 1,407 & 1,4358 & -0,593 & 2,04\end{array}$

cuivre à partir des données sur la compressibilité dynamique de ces métaux [12].

La figure 6 reproduit les courbes de Hugoniot des composés $\mathrm{Al}-\mathrm{Cu}$ calculées suivant la méthode indiquée ci-dessus, ainsi que nos points expérimentaux. L'accord entre valeurs expérimentales et valeurs théoriques paraît très satisfaisant. Les hypothèses du calcul concernant cette méthode semblent donc applicables aux types de composés que nous avons étudiés, en particulier la stabilité du choc, que nous avons vérifiée précédemment, et l'assimilation du composé à un corps homogène isotrope.

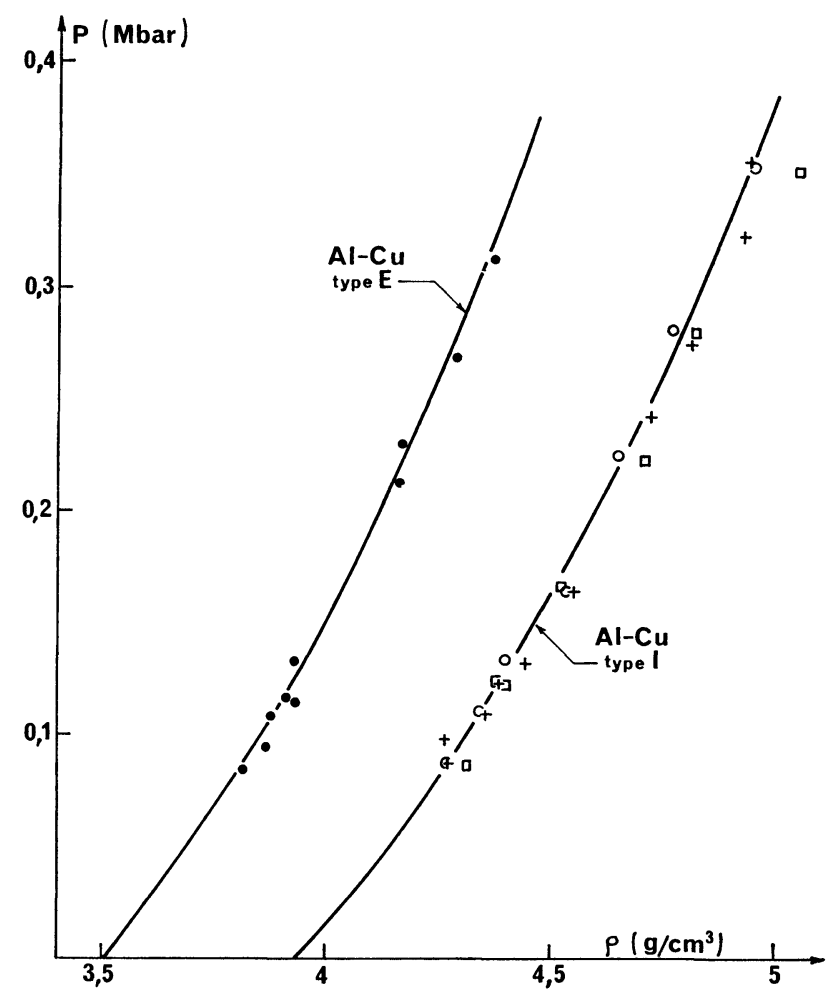

Fig. 6. - Comparaison des courbes de Hugoniot théoriques aux résultats expérimentaux : Résultats expérimentaux : $\bigcirc$ type $\mathrm{E}$, + type $I_{1}, O$ type $I_{2}, \square$ type $I_{3}$. - Courbes calculées selon le modèle adiabatique de Tsou et Chou [1].

5. Conclusion. - Nous avons pu mettre en évidence, grâce à une méthode de détection originale l'existence d'un choc stable dans différents types de composés lamellaires $\mathrm{Al}-\mathrm{Cu}$, ce qui nous a conduits à assimiler ces composés, dans leurs comportement sous choc, à des milieux homogènes isotropes. L'existence d'une relation linéaire entre vitesse de choc et vitesse particulaire ainsi que la comparaison satisfaisante entre les courbes de Hugoniot expérimentales et les courbes calculées selon le modèle adiabatique de Tsou et Chou ont confirmé ce résultat.

L'analogie de comportement entre ces milieux composites lamellaires et les milieux homogènes est un résultat qui s'inscrit dans le cadre plus large de l'étude des matériaux hétérogènes en général. Nous 
avons pu voir que l'épaisseur des lamelles ne constitue pas un facteur déterminant sur les propriétés de compressibilité dynamique des matériaux étudiés. Dans la mesure où seule la composition globale intervient, les structures lamellaires pourraient constituer des modèles pratiques pour l'étude des matériaux composites de structure plus compliquée, voire les alliages métalliques par exemple.

Remerciements. - La réalisation particulièrement délicate des montages a été assurée par J. C. Giraud que nous tenons à remercier ici.

\section{Bibliographie}

[1] Tsou, F. K. and Chou, P. C., J. Composite Materials 4 (1970) 526.

[2] Zababakhin, E. I., Sov. Phys. JetP 22 (1966) 446.

[3] Kozyrev, A. S., Kostyleva, B. E. and Ryazanov, V. T., Sov. Phys. JETP 29 (1969) 233.

[4] Torvik, P. J., J. Composite Materials 4 (1970) 296.

[5] McQueen, R. G., Marsh, S. P., TaYlor, J. W., Fritz, J. N. and CARTER, W. J., High Velocity impact phenomena (R. Kinslow), (Academic Press) 1970.

[6] BARKer, L. M., J. Composite Materials 5 (1971) 140.

[7] Garg, S. K. and Kirsch, J. W., J. Composite Materials 5 (1971) 428.

[8] Holmes, B. S. and Tsou, F. K., J. Appl. Phys. 43 (1972) 957.
[9] JACQUESSON, J., Coll. International CNRS no 109 , Les Ondes de détonation (édit. CNRS) (1962) 415.

[10] Crosnier, J., Jacquesson, J., Migault, A., Fourth symposium on detonation, White Oak, Silver Spring, Maryland (USA) (1965) 627.

[11] Migault, A., Thèse no 106, Poitiers (mai 1970).

[12] Rice, M. H., McQueen, R. G. and Walsh, J. M., Sol. St. Phys. (1958) 1.

[13] Van Thiel, M., Compendium of shock wave data, UCRL 50108, Lawrence Radiation Laboratory, University of California (June 1966).

[14] Berger, J. et Viard, J., Physique des explosifs solides (Dunod Paris) 1962.

[15] Gschneidner, K. A., Sol. St. Phys. 16 (1964) 275. 